\title{
Erratum: Selective contacts drive charge extraction in quantum dot solids via asymmetry in carrier transfer kinetics
}

Ivan Mora-Sero, Luca Bertoluzzi, Victoria Gonzalez-Pedro, Sixto Gimenez, Francisco Fabregat-Santiago, Kyle W. Kemp, Edward H. Sargent \& Juan Bisquert

Nature Communications 4:2272 doi: 10.1038/ncomms3272 (2013); Published 12 Aug 2013; Updated 30 Sep 2013 ; Updated 22 Nov 2013

Typographical errors were inadvertently introduced into equations (3)-(6) during the production process, such that the term $T$, representing temperature, is missing from the denominator of some exponents. These errors do not affect the analysis of results presented in the paper. The correct equations follow.

$$
\begin{gathered}
n=N_{\mathrm{c}} e^{\frac{E_{\mathrm{Fn}}-E_{\mathrm{c}}}{k_{\mathrm{B}} T}}=n_{0} e^{-\frac{q V_{\mathrm{n}}}{k_{\mathrm{B}} T}} \\
p=N_{\mathrm{v}} e^{\frac{E_{\mathrm{v}}-E_{\mathrm{Fp}}}{k_{\mathrm{B}} T}}=p_{0} e^{-\frac{q V_{\mathrm{p}}}{k_{\mathrm{B}} T}} \\
j_{m / \mathrm{QD}}^{n}=j_{\mathrm{rec}, n}^{s}\left(e^{\left(\beta_{n}-1\right) \frac{E_{\mathrm{Fm}}-E_{\mathrm{Fn}}}{k_{\mathrm{B}} T}}-e^{\beta_{n} \frac{E_{\mathrm{Fm}}-E_{\mathrm{Fn}}}{k_{\mathrm{B}} T}}\right) \\
j_{m / \mathrm{QD}}^{p}=j_{\mathrm{rec}, p}^{s}\left(e^{\beta_{p} \frac{E_{\mathrm{Fp}}-E_{\mathrm{Fm}}}{k_{\mathrm{B}} T}}-e^{\left(\beta_{p}-1\right) \frac{E_{\mathrm{Fp}}-E_{\mathrm{Fm}}}{k_{\mathrm{B}} T}}\right)
\end{gathered}
$$

